\title{
A Brief Analysis on the Application of Listening Strategies in Japanese Teaching
}

\author{
Yan Zhang \\ Japanese Dept. Dalian Neusoft University of Infor mation, Dalian, Liaoning, China
}

\begin{abstract}
Based on the results of students' listening strategies questionnaires, the thesis aims to acquire how current Japanese-majored students perceive and apply listening strategies, analyze the existing questions in listening teaching and find out students' specific obstacles in Japanese listening. On the basis of listening strategy theory and classification, the thesis adopts the 4-step listening cognitive strategy (predicting, note taking, applying and summarizing). Taking one teaching practice for example, the thesis studies the application of listening strategies in Japanese teaching through the 3 steps of pre-listening, listening and post-listening.
\end{abstract}

Keywords - listening strategies, Japanese teaching, application

\section{浅析听力策略在日语教学中的应用}

\author{
张岩 \\ 大连东软信息学院日语系, 大连, 辽宁, 中国
}

摘 要 本文基于学生的听力策略问卷调查, 了解目前日语专业学生对听力策略的认识及使用情况, 分析现阶段听力课存在的问题 及学生听力障碍的具体原因。此后, 基于听力策略理论及分类, 结合问卷调查, 选定听力认知策略中的预测、记笔记、演绎应用、总 结归纳 4 项听力策略。以一次听力教学实践为例, 通过听前、听中、听后三个阶段, 探讨听力策略在日语教学中的应用。

关键词 听力策略, 日语教学, 应用

\section{1. 引言}

21 世纪以来, 随着中日经济交流的不断扩大，市场对 日语人才的需求量不断增加, 对日语人才听说能力的培养 变得尤为重要。但听力课程授课效果不佳、学生听力水平 低却是一个不争的事实。在听力教学研究方面英语较为领 先, 且听力策略的研究一直是关注的重点。以苏远连 (2003)、朱湘华 (2013) 为代表, 很多学者针对听力策略 训练的模式和效果进行了研究。日语听力相关研究较少, 且研究主要局限在听力理解影响因素、听力应试等方面。 日本的及川浩二 (2012) 针对日语留学生考察了听力策略 训练的效果。在国内, 1994-2014 年的中国期刊全文数据库 中, 仅有聂星超（2013）的《多媒体听力策略对提高词汇 量的效能及调查分析》一篇文章与听力策略有关, 且重点 考察听力策略对词汇附带习得的影响。

本文拟在听力策略问卷调查的基础上, 了解学生对听
力策略的认识和使用情况。根据听力策略理论和分类, 选 定受训的听力策略, 尝试听力策略在日语教学中的应用。

\section{2. 听力策略问卷调查}

\section{1 调查概要}

为了解学生对听力策略的认识和听力策略使用情况, 笔者针对本校日语专业二年级 30 名学生进行了问卷调查。 问卷内容主要包括: “对听力策略的认识”, “听力策略使用 情况, 听力策略是否有效果”, “你认为现阶段最大的听力 障碍是什么?”3 大问题。

\section{2 听力策略问卷调查结果}

调查结果显示, 约 $60 \%$ 的学生不知道听力策略的内涵,

本文系 2014 年度辽宁省普通高等教育本科教学改革研究立项项目 (辽教发[2014]169 号)《应用型本科日语专业人才培养质量标准体系 的研究与实践》成果之一 
约 $40 \%$ 学生认为听力策略是一种方法, 还有学生认为所谓 听力策略就是多听、多背单词。针对听力策略的内容, 有 $70 \%$ 的学生指出了具体的听力方法, 例如听关键词、做笔 记、听前先看试卷和图、了解相关信息, 注意まず、それ から、やっぱり等功能词后项的内容等。约 $80 \%$ 的学生认 为曾经使用过听力策略 (听力方法), 听力策略训练对于提 高日语听解能力应该有一定的效果。最后一项, 学生针对 目前最大的听力障碍的认识虽然不尽相同, 但约有 $80 \%$ 学 生提出日语基础知识薄弱是最主要的问题。由于音频资料 语速较快, 听后单词、语法反映不过来, 影响了听力效果。 还有学生提出听力练习较少, 没有掌握听力方法等问题。

\section{3 现阶段听力课存在的问题及学生听力障碍的具体原因}

由于听力理解是在学生大脑内部发生的学习过程, 所 以“听”与说和写相比具有很强的内隐性。一直以来, 听力 理解多被看成是一个被动的、接受性的过程。现阶段, 听 力课仍然沿袭传统的授课模式, 注重单词、语法的讲解, 认为日语水平提高则听解能力就自然而然的提高了。提高 听力水平只能通过反复的听说练习来实现, 忽视了策略训 练的重要性。这一教学模式和思想直接影响了学生对听力 课和听力障碍的认知。

日语基础知识确实是影响听力水平最主要的因素, 但 听力策略能在一定程度上提高听解效果, 起到锦上添花的 辅助作用。并且通过问卷调查可以看出学生已经了解并尝 试使用部分听力策略, 但还处于无意识的、模糊的状态, 具体策略的运用方法还有待教师通过教学实践给予明确的 指导, 以提高使用听力策略的效果。

\section{3. 听力策略理论及分类}

\section{1 听力策略理论}

听力策略作为 “语言学习策略”的一部分, 其理论依据 和框架与“语言学习策略”一致。Rubin(1987)把学习策略概 括为学生为成功获取、咜存、再获取并使用语言信息而采 取的操作步骤、计划和管理行为。Oxford(1990)认为, 学生 的策略是指学生在语言学习过程中采取的具体行动, 以使 语言学习变得更加轻松、快捷、有趣、有效, 并适用于新 环境下的学习。笔者认为听力策略, 是指学习者在听力理 解的过程中, 采用具体的方法或手段, 听懂并掌握音频内 容的思想和行为。听力理解过程是一个积极建构意义的过 程, 策略的运用对信息的笁选、处理、记忆、储存和提取都 起着举足轻重的作用。

\section{1 听力策略的分类}

朱湘华以 O 'Malley\&Chamot(1990)的框架为蓝本, 应 用 Oxford(1990)和文秋芳(1995)的部分策略, 结合听力理解 过程的特点, 将听力策略归纳为元认知策略、认知策略和社 会情感策略三大策略。元认知策略是一种高级的执行性技 巧、涉及学生为促进某一学习活动 (比如理解语言) 的顺 利完成而进行的计划、监控、评估等行动。认知策略涉及 对输入信息的处理, 包括再现/重述、总结、推理以及细化 等策略。社会情感策略涉及学生为完成某一学习任务而跟 别人进行交流或自己控制情绪、消除不安后疑惑等策略。

\section{4. 听力策略在日语教学中的应用}

\section{1 选定训练策略，制定策略训练计划}

三大策略中认知策略与具体的语言听力任务有关, 是 学生利用日语固有的基础知识等对输入的信息进行加工处 理时所采用的特定技巧。在教学实践中, 可操作性强。因 此本文选定认知策略中预测、记笔记、演绎应用、总结归 纳 4 项, 以一次听力教学实践为例, 通过听力听前、听中、 听后三个阶段, 尝试在日语教学中进行听力策略训练。

\section{2 听力策略在日语教学中的应用}

基于二年级下学期日语专业学生的听力水平, 选定由 大连理工出版社出版的《中级日语听力教程 (上)》第 44 课《イヌ人間とネコ人間》为音频资料。

听前, 首先让学生根据教师的提问, 预测可能听到的 内容。例如教师可以提问如下问题:

ペットを飼うことがあります。

イヌとネコとどちらが好きですか。どうしてですか。

イヌとネコはそれぞれどんな性質を持っています。

学生思考后首先对本课的听力材料产生了兴趣, 并可 能就狗和猫的特质进行简要的分析。例如: 狗很忠诚、注 重集体的团队; 而猫则喜欢偷懒, 爱玩。

听中, 让学生听第一遍录音, 明确「イ又人間」和「六 ב人間」的特点。基于听前预测, 则不难听懂如下内容 (「イ 又人間は勤勉で、集団の利益を重んじてるのに対して、 ネコ人間は怠け者、遊び好きで、個人の利益を求めます」)。 但是对于为什么会出现「イ邓人間」和「ネコ人間」的提 法, 由于前半段音频数字较多, 需要再听一遍录音, 通过 记笔记的方式来理清思路、确认内容。

记笔记时, 由于录音速度较快, 如果记录听到的所有 信息反而会导致只注意了细枝末节, 而忽略了文章的大意。 因此, 教师要积极教授学生用缩写、图表或数字的形式记 
录关键词语或概念。例如针对本音频材料(余㗇時間は 1975 年からかえって減り続けて、1988 年にはアメリカおり約 $20 \% 、 フ ラ ン ス ょ り$ 約 $30 \%$ も少なくっています), 学生需 要记录时间。笔者建议可以指导学生采用如下方式记录。

例如: 余 1977-,1988 ア 20\%-, > 30\%-。

演绎应用策略是指利用所学的词、句法等语言知识推 断生词、句子意思, 如利用同源词、接续词、副词等。本 音频材料中仅有「犠牲」一个生词。音频原文为: ですか ら、日本のサラリーマン社会は個人の時間を犠牲にして、 会社のために働いてきたというわけです。接续词「です から」, 表明前后文为因果关系。「犠牲」(ぎせい)与汉语发 音相近, 通过前后句的意思, 不难推测此单词的意思。

听后, 多采用总结概括听力策略。通过读听力材料音 频、用日语总结音频资料大大意等活动, 让学生巩固所听 到的话题内容。并让学生尝试谈论生活动类似的事件、阅 读相关的材料、扩展相关知识。例如本课可以让学生谈论 一下日本的「余暇生活」和「残業文化」, 以了解日本文化、 扩展日本企业文化相关知识。最后通过策略评估问卷反馈 的方式, 让学生对策略的使用情况进行回顾, 并评价策略 使用后的效果。

\section{5. 结语}

如上, 笔者以一次听力课程实践为例, 探讨了听力策 略在教学中的应用。听力策略训练在一定程度上能够起到
辅助教学, 提高听力水平的作用, 但学生的日语基础知识 是影响听力水平最重要的因素, 不容忽视。而且听力策略 训练、学生听力意识的改变、听力水平的提高是一个长期 的过程。本文仅对听力策略训练中短期的、分离式、隐形 的训练模式进行了教学尝试, 今后会有计划的开展长期、 融合式、显性的听力策略训练模式。同时尝试进行量性的 实证研究, 以考察听力策略训练对于提高日语听解水平的 效果。

\section{参考文献(References)}

[1] Oikawa Hirokazu, Kato Naoki, "The Analysis of Learning effect by Instruction Training of Hearing Strategy for Foreign Students," Japan Society of Educational Information, vol.29(1), pp.13-19, September 2013.

[2] Qingmei Ren, "English listening study, "Foreign Language Teaching and Research Press, August 2011.

[3] Xianghua Zhu, “ Listening strategies in meta cognitive awareness and the analysis of intermediary effect between English listening scores, "Foreign Language World, vol. 8, pp. 53-58,2013.

[4] Zidong Huang, “Western second language/foreign language listening comprehension strategies research review, "Foreign Language World, pp. 43-47,1998. 